\title{
LA EVALUACIÓN CALIDAD-PRECIO EN LA TRIANGULACIÓN ENTRE SATISFACCIÓN, RECOMENDACIÓN Y LEALTAD DEL VISITANTE INTERNACIONAL TERRESTRE A BAJA CALIFORNIA, MÉXICO
}

\author{
Djamel Toudert \\ Nora L. Bringas Rábago \\ El Colegio de la Frontera Norte. México
}

\section{RESUMEN}

El objetivo principal de este ensayo consta en examinar la pertinencia de la evaluación calidad-precio como antecedente o hecho posterior a la satisfacción turística, así como sus impactos en la lealtad del visitante y su disposición a recomendar el destino. Esto procedió por medio de dos modelos de investigación sustentados en datos de visitantes internacionales terrestres a Baja California, México. En este destino, además de la importancia de la satisfacción para la competitividad, la percepción positiva del costo por un visitante satisfecho permite también reconsiderar el precio a favor de la oferta. Esta investigación confirma la significación de la calidad-precio en ambos modelos. En el primero, se cumple mejor con un propósito exploratorio siendo la valoración calidad-precio antecedente de la satisfacción, mientras que el segundo parece adecuarse a un fin predictivo cuando la calidad-precio es consecuencia de la satisfacción.

Palabras clave: Evaluación calidad-precio; satisfacción turística; recomendación y lealtad; visitantes internacionales; Baja California.

Recibido: 22 de julio de 2014

Devuelto para su revisión: 19 de noviembre de 2014

Aceptado: 16 de diciembre de 2014

Departamento de Estudios Urbanos y Medio Ambiente. El Colegio de la Frontera Norte. Carretera Escénica Tijuana-Ensenada, Km 18.5. San Antonio del Mar. 22560 Tijuana. BAJA CALIFORNIA. México. E-mail: toudert@colef.mx y nbringas@colef.mx 


\title{
Price-quality evaluation in the triangulation of satisfaction, recommendation, and loyalty of international travelers arriving by land to Baja California, Mexico
}

\begin{abstract}
The main objective of this paper is to examine the relevance of the price-quality assessment as an antecedent or subsequent event to tourist satisfaction, as well as their impact on visitor loyalty and the possibility to recommend the destination. It was done through two research models supported with data from land international visitors to Baja California, Mexico. At this destination, besides the importance of satisfaction for competitiveness, the positive perception of cost per satisfied visitor also allows to reconsider the price in favor of the offer. This research confirms the significance of price-quality in both models. In the first model, an exploratory purpose is best fulfilled having the price-quality assessment as an antecedent to satisfaction, while the second model seems to fit a predictive goal when the value is a consequence of satisfaction.
\end{abstract}

Keywords: Price-quality evaluation; tourist satisfaction; recommendation and loyalty; international travelers; Baja California.

\section{INTRODUCCIÓN}

El conocimiento de la satisfacción del visitante para los procesos de toma de decisiones en el campo de la gestión y mercadotecnia se ha convertido en un aspecto estratégico para el desarrollo de la actividad turística en todos sus niveles (Bigné et al., 2001; Bigné, y Andreu, 2004; Chen y Chen, 2009). En este contexto, tanto las variables que anteceden a la satisfacción turística, como la calidad-precio, o las que se desprenden de ella, como la recomendación y la lealtad al destino, se han vuelto sujetos de estudio y de aplicación para maximizar el consumo repetitivo de los productos turísticos (Yoon y Uysal, 2005; Yuksel et al., 2009).

Varios trabajos orientados a explicar la importancia de la satisfacción turística coinciden en subrayar su impacto anticipatorio en la recomendación del destino y en el fortalecimiento de la lealtad hacia el consumo (Yoon y Uysal, 2005; Chi y Qu, 2008; Yuksel et al., 2009). Una vez trasladados estos aspectos a la gestión turística, esta última se ha visto reflejada en una constante reducción del costo asociado con la captación de nuevos visitantes, traduciéndose en una creciente rentabilidad de la empresa y/o del destino (Baker y Crompton, 2000; Petrick, 2004).

Del lado de la conceptualización de la satisfacción del consumidor, los pocos trabajos que se enfocaron al estudio del impacto de la calidad percibida, reflejada en el presente estudio por la calidad-precio, hallaron un impacto positivo en la satisfacción (Cronin, et al., 2000; Rust, et al., 2000; Pedraja y Yagüe, 2004). Además resulta quizás necesario mencionar que estas aproximaciones enfocaron a la calidad-precio, principalmente, como antecedente de la satisfacción turística a pesar de que no existe, como se aclarara más adelante, un impedimento conceptual para abordarla en el marco de un constructo posterior a la satisfacción del visitante. No obstante, independientemente 
de la trascendencia y versatilidad de la calidad percibida en el consumo, su estudio no parece beneficiarse de un amplio examen por la investigación empírica (Zeithmal, 1988; Dodds, et al., 1991; Voss et al., 1998). Es en el marco de esta necesidad de ampliar los intereses exploratorios que este trabajo se fija el objetivo de averiguar y comparar la pertinencia estadística de un planteamiento reciproco que concibe, en un primer modelo de investigación, a la calidad-precio como precedente de la satisfacción turística y, en un otro modelo, como su posible resultado. Lo anterior tiene como contexto de estudio la evaluación de la estancia del visitante internacional terrestre a los destinos de Baja California, México que comparten con otros similares a una situación de escasez de recursos para enfrentar las problemáticas de desarrollo derivadas -entre otros- del crecimiento de la actividad turística (Bringas Rábago y Verduzco Chávez, 2008). En estos entornos visitados, independientemente de la importancia de la satisfacción del visitante para incrementar la demanda, de resultar determinante en la valoración positiva de los precios pagados durante la estancia, se estaría abriendo también la posibilidad para una revisión del precio a favor de los actores de la oferta.

La exploración de las relaciones causales entre la evaluación de la satisfacción turística y la calidad-precio se sustenta en la elaboración de dos diseños de investigación (modelo A y B) que divergen únicamente por la manera de incorporar la evaluación calidad-precio (véase figura 2). Aunque estos dos modelos de investigación parecerían expresar situaciones semejantes, sus lógicas de involucrar a la calidad-precio los hacen diferentes y susceptibles de reflexiones con consecuencias potencialmente disparejas (Véanse trabajos de Churchill y Surprenant, 1982; Fornell, 1992; Halstead et al., 1994).

En el modelo A la evaluación calidad-precio interviene como un constructo inherente a la satisfacción turística, con un impacto en el incremento del flujo de visitantes por medio de la recomendación y la lealtad del consumo. Mientras en el modelo B, la satisfacción turística se afirma como antecedente a la evaluación de la calidad-precio en un planteamiento que posibilita la reconsideración del precio en una relación favorable a la oferta. Este último aspecto puede resultar sugestivo para una mayor contribución del turismo al desarrollo del destino, sin embargo profundizar en estos tópicos requiere por sí mismo de una extensión que va más allá de lo designado para este ensayo.

En el marco del presente trabajo, el planteamiento conceptual de ambos modelos de investigación se centra en la perspectiva de la satisfacción que se desprende de la relación entre los costos pagados y los beneficios recibidos, una vinculación conocida por desembocar a menudo en una ambigüedad de lecturas (Halstead et al., 1994).

Para evitar las confusiones de interpretación que suelen desprenderse de la dualidad de la relación entre la satisfacción del consumidor y los componentes de la utilidad percibida (calidad percibida y sacrificio consentido), esta última tomará la expresión de la calidad percibida en el modelo A (Zeithmal, 1988; Dodds et al., 1991; Petrick, 2004; Ainscough, 2005). En efecto, como se señala en los trabajos de Cronin y Taylor (1992); Rust et al. (2000); Baker y Crompton (2000), el incremento en la evaluación de la calidad percibida se traduce en un aumento de la satisfacción, mientras que a mayor percepción del sacrificio consentido corresponde una menor satisfacción del consumidor (Dodds, et al., 1991; Pedraja y Yagüe, 2004). Estas mismas confusiones de interpretación se encuentran reflejadas, conforme a lo reportado por Rao 
y Monroe (1989) y Campo y Yagüe (2009), en la duplicidad de la evaluación de la calidad que abarca conceptualmente los precios de referentes internos conformados por estímulos y experiencias personales, y precios de referentes externos estructurados por variables del contexto de venta (Yadav y Seiders, 1998; Moon, et al., 2006).

En este sentido, la calidad de un producto y/o servicio puede enfocarse desde la perspectiva de sus atributos intrínsecos (referentes inherentes) y/o por medio de sus referentes exógenos que expresan sus variables contextuales (Rao y Monroe, 1989; Campo y Yagüe, 2009). Bajo esta perspectiva, el modelo A estará expresando la calidad percibida encapsulada como referente inherente de la satisfacción turística, mientras que el modelo B centra su interés en la calidad percibida como un referente exógeno, es decir, un constructo determinado a partir de la satisfacción turística que se encuentra definida a su vez por sus constructos predictivos en el modelo de investigación.

\section{Figura 1}

\section{UBICACIÓN DE LA REGIÓN DE ESTUDIO}

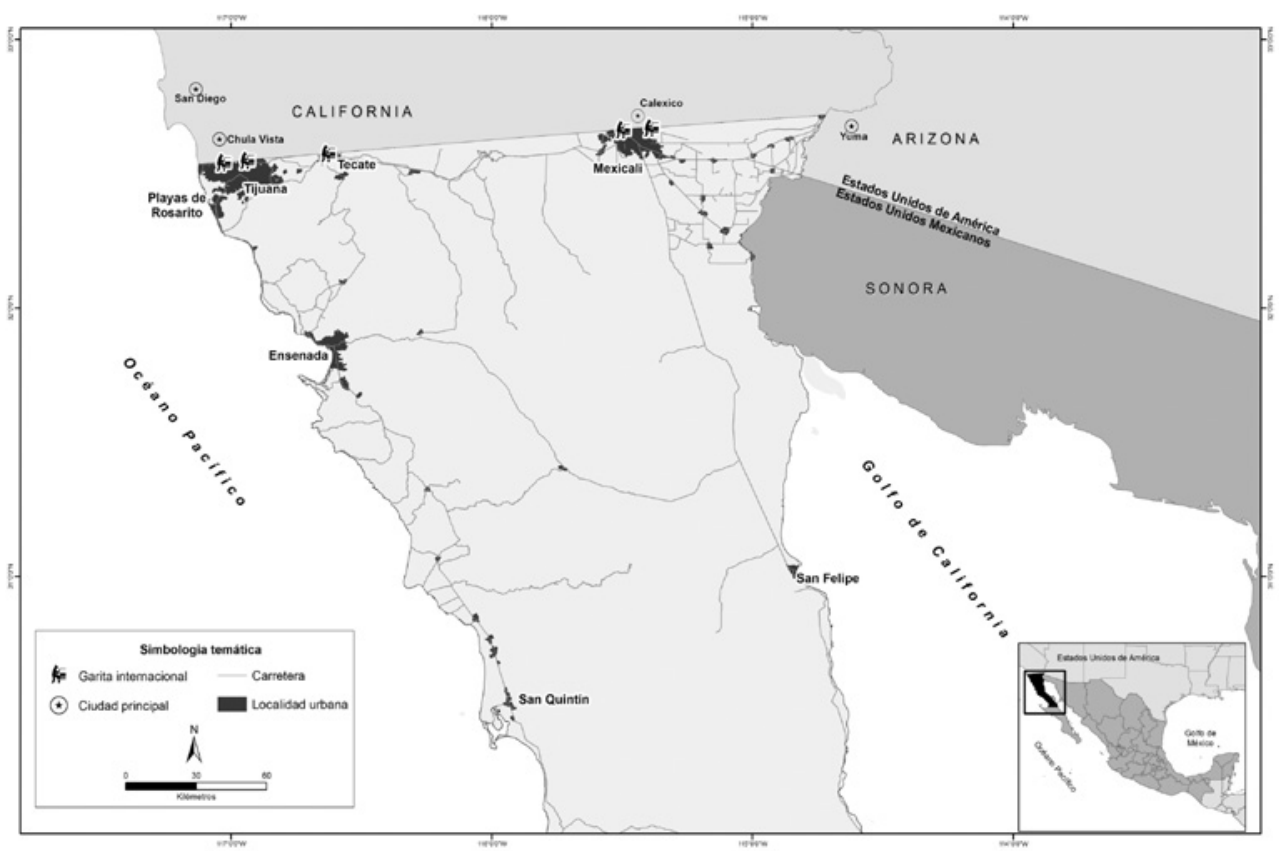

Fuente: Elaboración propia.

En su conjunto, este trabajo fue sustentado en datos de la encuesta de verano de 2013 (OTBC, 2013), aplicada por el Observatorio Turístico de Baja California (OTBC) en el marco de un estudio amplio de la demanda turística internacional terrestre (que incluye principalmente el perfil sociodemográfico, características de la estancia, consumo de bienes y servicios turísticos y satisfacción del visitante). En este sentido, aunque la iniciativa del OTBC posibilitó beneficiarse de un marco comparativo con la modelación de la 
satisfacción turística de CESTUR (2011), cabe aclarar que los intereses epistemológicos de esta investigación no fueron los propósitos exclusivos de la encuesta aplicada.

De esta manera, para cumplir con los objetivos planteados el presente trabajo se estructuró de la siguiente forma: La sección 2 aclara los alcances teórico-conceptuales de esta investigación; la sección 3 describe y analiza el eje informacional, el desarrollo metodológico e instrumental; y por último, se revelan y discuten los hallazgos de este trabajo.

\section{POSICIONAMIENTO TEÓRICO-CONCEPTUAL DE LA INVESTIGACIÓN}

La investigación sobre la satisfacción turística y las variables asociadas con la misma parece enfrascada, quizás por su naturaleza abstracta y multidimensional, en una amplitud de intereses epistemológicos y conceptuales que no han facilitado la búsqueda de un matiz teórico sólido y aún menos, logrado un marco unificado (Wang et al., 2014).

Como punto de reflexión inicial, el presente trabajo parte de la adaptación del modelo de investigación de la satisfacción turística desarrollada por CESTUR (2011) sumándole la dimensión que se desprende de la lealtad del visitante respecto al destino (LEAL) y desagregando los servicios públicos (SERP) o privados (SERPAR) que utiliza el turista. La integración de la lealtad interviene en el marco de una consolidación conceptual del modelo de investigación tomando en cuenta los trabajos que abogan en esta dirección (Bigné et al., 2001; Bigné y Andreu, 2004; Yoon y Uysal, 2005; Chi y Qu, 2008; Yuksel et al., 2009), mientras que la desagregación de los servicios obedece a una adecuación contextual del tipo de turismo analizado (OTBC, 2013).

La satisfacción turística que se identifica generalmente con la dimensión cognitiva de las percepciones y emociones caracteriza uno de los temas más explorados por la investigación turística (Bosque y Martin, 2008; Hasegawa, 2010). Independientemente de si no ha logrado todavía un marco conceptual unificado, la satisfacción turística es considerada un aspecto central de la estrategia de mercadeo, destacándose por un impacto recíproco y positivo sobre las ventas y la consolidación de la cultura de la satisfacción en el seno de la organización (Fornell, 1992). Estas interconexiones positivas son estimuladas también por la lealtad del consumidor que motiva la recomendación a conocidos, traducida en la captación -sin costos aparentes para la organización-, de nuevos clientes (Söderlund, 1998; Chi y Qu, 2008). De hecho, algunos autores consideran pertinente el ahorro generado con la conservación de un cliente satisfecho, comparado con el costo que implica atraer a uno nuevo (Fornell y Wernerfelt, 1987; Reichheld, 1996). En este sentido Gupta et al. (2004) observaron que un incremento del $1 \%$ en la retención de consumidores se traduce en una ganancia del $5 \%$. Además, se cuenta con evidencias de un costo asociado menor con la atracción de un cliente recomendado por un consumidor satisfecho comparado con el gasto de captación de un nuevo cliente por medio de estrategias de mercadeo (Shoemaker y Lewis, 1999; Alegre y Juaneda, 2006).

En el marco de la triangulación conceptual entre satisfacción del visitante, lealtad al destino y disposición a recomendarlo a conocidos, la lealtad alcanza un doble significado fundamentado en un comportamiento actitudinal favorable al contexto visitado y la propensión a volver a visitarlo en repetidas ocasiones (Oppermann, 2000; Chen y Chen, 
2009). Este tipo de integración que llega a conjugar la repetición de la compra con la actitud de promocionar lo adquirido, se desprende de la satisfacción del turista que les proporciona audacia y fortaleza para su desenvolvimiento (Kozak y Rimmington, 2000; Bigné et al., 2001; Yoon y Uysal, 2005; Chi y Qu, 2008).

Desde la perspectiva de las intenciones de comportamiento, la recomendación a conocidos y la satisfacción con la calidad percibida son dimensiones influidas por la comunicación de «boca a oreja», propósitos de compra y la sensibilidad a los precios (Zeithaml et al., 1996; Kim et al., 2009). Esta última, traduce la disposición del consumidor a gastar más cuando su satisfacción es menos sensible al costo, pero vinculada con el precio de lealtad (Yoo y Park, 2007). En este sentido, si el costo no es el elemento central de la toma de decisión de compra, los consumidores suelen presentar mayor tolerancia a los precios cuando confían y se encuentran satisfechos con lo ofertado (Reichheld y Sasser, 1990). En el marco de esta perspectiva, adquiere viabilidad conceptual el modelo de investigación B cuando plantea una relación positiva desde la satisfacción turística hacia la evaluación calidad-precio. Mientras en el modelo de investigación A, la sensibilidad a los precios queda hipotéticamente manifiesta en el marco de una relación multifacética antecedente a la satisfacción turística.

Conjuntamente a la vinculación de la calidad-precio con la satisfacción vista como un constructo multidimensional, la accesibilidad del destino y la percepción que se desprende de la prestación de servicios públicos, constituyen aspectos fundamentales tanto en la reflexión como en la acción alrededor de la actividad turística (González Fernández y Esteban Talaya, 2000; Wickens, 2002; Chi y Qu, 2008). Lo anterior cobra una posición central en el caso del turismo internacional fronterizo, que se caracteriza por su sensibilidad a las cuestiones de seguridad pública y personal (Bringas Rábago y Verduzco Chávez, 2008).

El mismo tipo de relaciones causales entretejidas alrededor de la satisfacción turística fueron observadas también en lo relativo a las prestaciones de hospedaje (Bonet Agustí, 2008; Albacete-Saez et al., 2007), alimentación y bebidas (Elías Pastor, 2006; Chi y Qu, 2008) y actividades recreativas (González Fernández y Esteban Talaya, 2000; Bonet Agustí, 2008). En el marco de estas evidencias, Castaño et al. (2003) confirmaron que la actitud social en el destino influye en la satisfacción y la lealtad turística; por cierto, esta última constituye una dimensión menos influida por la motivación del viaje (Gundersen y Olsson, 1996; Laguna y Palacios, 2009). Estas afirmaciones ubican a la conceptualización de la satisfacción en un contexto socioterritorial amplio comparado con el entorno inmediato de interacción del turista (Devesa y Palacios, 2005), un detalle teórico que parece llevar la modelación empírica a otros niveles de complejidad.

\section{DATOS Y METODOLOGÍA DE INTEGRACIÓN DE LA INFORMACIÓN}

Tomando en cuenta la calidad multidimensional y la complejidad estructural de los dos modelos de investigación planteados en este trabajo, resulta apropiado optar por la aplicación de técnicas de regresiones múltiples que permiten analizar las relaciones causales entre los diferentes constructos de la satisfacción (Burnette y Williams, 2005). En el marco de esta elección, la falta de un campo teórico sólido, la carencia de normalidad en los datos analizados y el involucramiento de constructos informativos, conllevaron a 
adoptar un método exploratorio predictivo orientado a la construcción de la teoría como lo es la técnica de los mínimos cuadrados parciales, conocida por sus siglas en inglés por PLS: Partial Leaste Squares (Chin, 1998; Hsu et al., 2006).

La modelación PLS se sustenta en modelos reflexivos y formativos que se construyen por medio de las variables manifiestas o ítems de la evaluación realizada a cada concepto de la satisfacción turística (véase figura 2 y cuadro 1). En este sentido, el modelo interno formado por las variables latentes (o constructos) y el modelo externo estructurado por las relaciones entre las variables manifiestas y los constructos, son los elementos a examinar para caracterizar la validez y la solidez del modelo de investigación propuesto (Falk y Miller, 1992; Chin, 1998).

Los datos del presente trabajo provienen de la encuesta «Caracterización de los visitantes internacionales en las ciudades fronterizas de Baja California: Mexicali, Tecate y Tijuana» que fue levantada en los puertos fronterizos terrestre por el Observatorio Turístico de Baja California (OTBC) ${ }^{1}$ durante los meses de julio, agosto y septiembre del 2013.

La encuesta fue realizada con la aplicación de un muestreo estratificado y polietápico con probabilidades proporcionales que permitió lograr 2,704 cuestionarios completos con un margen de error teórico de $+/-5 \%$ para un nivel de confianza de 95\%, la corrección del efecto de diseño de $1.5 \%$ y una tasa de no respuestas estimada en un $15 \%$ (OTBC, 2013). El mismo número de cuestionarios fue involucrado en el presente estudio, destacando en el marco de los dos modelos de investigación un amplio cumplimiento con el tamaño de la muestra adecuada (Barclay et al., 1995) y las consideraciones críticas de Henseler et al., (2009) para el uso de la técnica PLS en un estudio exploratorio.

\subsection{Modelos teóricos y datos analizados}

Los modelos de investigación están compuestos en ambos casos por un grupo de 10 variables latentes, unas predictivas exógenas (ACCES, IMAGE, ATRACT, SERP, SERPAR, EXPER), otras endógenas (SATIS, RECO, LEAL) y la variable COSTO integrada como predictiva exógena en el modelo A y estimada endógenamente en el modelo B (véase figura 2). En su conjunto, las 10 variables latentes están vinculadas a 29 variables manifiestas (o Ítems) involucradas como indicadores reflectivos en el caso de las latentes ACCES, IMAGE, ATRACT, SERP, SERPAR, EXPER y STATIS e informativos para los constructos COSTO, RECO, y LEAL (Véase cuadro 1). La valoración por el entrevistado de estos ítems procede a partir de la aplicación de una escala de evaluación de la satisfacción oscilante entre 1 (pésimo) y 10 (excelente).

En el marco de estas características que definen el entorno operativo del estudio, se procedió se procedió con el cálculo del modelo de medidas y estructuras que serán analizados, más adelante.

1 El Observatorio Turístico de Baja California es un proyecto conjunto entre El Colegio de la Frontera Norte y la Secretaría de Turismo del Estado de Baja California, México. 
Figura 2

ILUSTRACIÓN DE LOS DOS MODELOS DE INVESTIGACIÓN

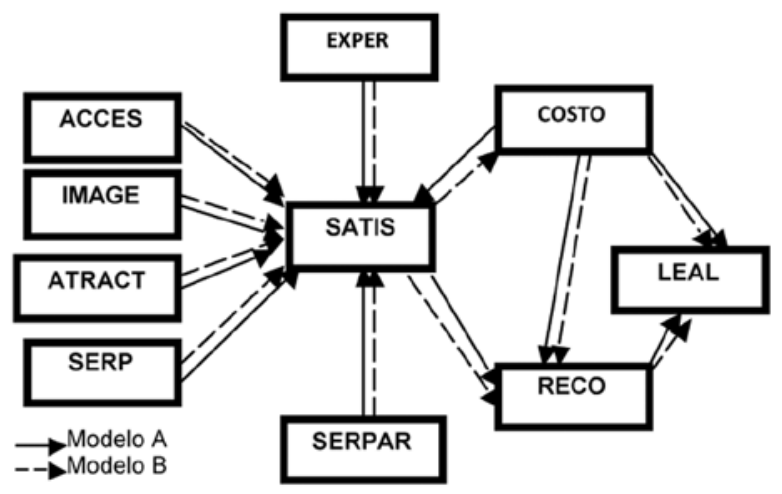

Fuente: Elaboración propia.

\section{Cuadro 1}

\section{VARIABLES LATENTES Y MANIFIESTAS INVOLUCRADAS EN EL ESTUDIO}

\begin{tabular}{|c|c|}
\hline ACCES- Accesibilidad del destino* & SERPAR- Servicios particulares* \\
\hline A1- Tiempo de espera para entrar a México & D2.5- Experiencia de consumo de alimentos y bebidas \\
\hline A2- Actitud del oficial de migración & $\begin{array}{l}\text { D2.6- Atención en los establecimientos de alimentos y } \\
\text { bebidas }\end{array}$ \\
\hline A3- Actitud del oficial de aduanas & $\begin{array}{l}\text { D2.7- Limpieza / higiene de los establecimientos de } \\
\text { alimentos y bebidas }\end{array}$ \\
\hline IMAGE- Imagen* & D2.8- Higiene en el manejo de los alimentos y bebidas \\
\hline B1- atractivos naturales y culturales & D2.9- Oferta gastronómica local \\
\hline B2- Limpieza de las calles y áreas públicas & EXPER- Experiencia de viaje* \\
\hline B3- Tráfico vehicular & E3- Emoción de visitar al destino \\
\hline B6- Imagen urbana & E4- Originalidad de la experiencia de viaje ofertada \\
\hline B7- Seguridad durante la visita & E5- Experiencia general de la visita \\
\hline ATRACT- Atractivos* & COSTO- Costo (informativo) \\
\hline C4- Estado de conservación de atractivos naturales & F1- Relación calidad-precio \\
\hline C5- Estado de conservación de playas & SATIS- Satisfacción* \\
\hline SERP-Servicios públicos* & G1- Satisfacción del viaje \\
\hline D1.1- Policía municipal & G2- Expectativa / satisfacción del viaje \\
\hline D1.2- Policía federal de caminos & G3- Satisfacción comparativa con visitas a otros destinos \\
\hline D1.3- Casetas de información turística & RECO- Recomendación** \\
\hline D1.4- Teléfonos públicos & H1- Recomendación del destino a conocidos \\
\hline \multirow[t]{2}{*}{ D1.5- Baños públicos } & LEAL- Lealtad** \\
\hline & I1- Disposición a volver a visitar el destino \\
\hline
\end{tabular}




\subsection{Pertinencia de los dos modelos analizados}

La estimación de la robustez de los dos modelos propuestos en esta investigación obedece a las pautas de evaluación de la modelación exploratoria con el uso de la técnica PLS (Barclay et al., 1995; Jörg et al., 2009). Bajo esta perspectiva, se analizará en primer lugar el modelo de medidas y posteriormente, la significación y alcance de las relaciones causales que encierren los modelos de investigación.

\subsubsection{Análisis del modelo de medidas}

En el marco del análisis del modelo de medidas se contempla llevar a cabo la evaluación de la fiabilidad de las variables latentes y sus ítems respectivos, además de una valoración de la validez convergente y discriminante.

Tanto en el modelo A como B, que comparten los mismos valores de carga de sus ítems, se destacan cifras superiores a la regla empírica admisible del 0.707 con una varianza observada (comunalidad) compartida por el constructo por encima de la tasa aceptable de 50\% (Nunnally y Bernstein, 1994; Chin 1998; Tenenhaus et al., 2005). Lo anterior se verifica en todos los casos salvo con la variable B1, que logra una carga muy cercana al valor aceptable, su conservación en el estudio obedece, tal como lo sugiere Chin (1998) a una relativa flexibilidad en la aplicación de la regla cuando la investigación se encuentra en sus estados iniciales.

En lo que corresponde a la evaluación de las variables manifiestas informativas de los constructos COSTO, RECO, LEAL, además de la validación nomológica generalmente se sugiere llevar a cabo mediciones de multicolinealidad, inspeccionar el peso de cada ítem, que debe ser superior a 0.1, examinar la validez externa de los constructos formativos y evaluar la validez discriminante de sus correlaciones (Bagozi, 1994; Diamontopoulos y Winklhofer, 2001; McKnight et al., 2002; Peng y Lai, 2012). En nuestro caso de estudio, los constructos informativos son conectados a un solo ítem y no incluyen variables reflectivas adicionales, ni constructos de tipo «sombra» que permiten llevar a cabo la evaluación de todas las pautas enumeradas anteriormente. Sin embargo esta situación se compensa por la empatía ítem-constructo y la validación nomológica reflejada en la alta significación de los constructos informativos con los demás constructos implicados en la investigación (véase cuadro 4).

Una vez establecida la fiabilidad de los ítems involucrados en el estudio, continuamos con la evaluación de la fiabilidad de las variables latentes reflectivas a través del análisis de la fiabilidad compuesta (CR) y los coeficientes alfa de Cronbach (AVE).

Con relación a la fiabilidad compuesta (CR), las variables latentes reflectivas presentan valores superiores a 0.8 reflejando un nivel de exigencia aceptable para una investigación básica (véase cuadro 3). De la misma manera, los coeficientes alfa de Cronbach (AVE) son más altos que el valor admisible establecido en un 0.5 (Chin, 1998; Tenenhaus et al., 2005). Estos valores de AVE que se reflejan en una tasa superior al $50 \%$ de la varianza que un constructo obtiene de sus variables manifiestas, confirman también el cumplimiento con la exigencia de la validez convergente del modelo de medidas (Fornell y Larcker, 1981). 


\section{Cuadro 2 \\ FIABILIDAD DE LOS ÍTEMS INVOLUCRADOS}

\begin{tabular}{|c|c|c|c|c|c|}
\hline Variables & Factor de carga & Comunalidad & Variables & $\begin{array}{c}\text { Factor de } \\
\text { carga }\end{array}$ & Comunalidad \\
\hline ACCES & & 0.8115 & SERPAR & & 0.7666 \\
\hline A1 & 0.8282 & & D2.5 & 0.911 & \\
\hline A2 & 0.9417 & & D2.6 & 0.82 & \\
\hline A3 & 0.9282 & & D2.7 & 0.8677 & \\
\hline IMAGE & & 0.5537 & D2.8 & 0.9155 & \\
\hline B1 & 0.6915 & & D2.9 & 0.8601 & \\
\hline B2 & 0.751 & & EXPER & & 0.7602 \\
\hline B3 & 0.7363 & & E1 & 0.8999 & \\
\hline B6 & 0.7836 & & E2 & 0.8255 & \\
\hline B7 & 0.7552 & & E3 & 0.8884 & \\
\hline ATRACT & & 0.7721 & SATIS & & 0.8698 \\
\hline C4 & 0.9243 & & G1 & 0.9446 & \\
\hline C5 & 0.8306 & & G2 & 0.9516 & \\
\hline SERP & & 0.5913 & G3 & 0.9009 & \\
\hline D1.1 & 0.7346 & & COSTO* & & \\
\hline D1.2 & 0.7594 & & RECO* & & \\
\hline D1.3 & 0.7278 & & LEAL* & & \\
\hline D1.4 & 0.8251 & & & & \\
\hline D1.5 & 0.7937 & & & & \\
\hline
\end{tabular}

${ }^{*}$ Constructos informativos de un solo ítem.

Fuente: Elaboración propia.

\section{Cuadro 3}

\section{VALIDEZ CONVERGENTE Y DISCRIMINANTE}

\begin{tabular}{|c|c|c|c|c|c|c|c|c|c|c|c|c|}
\hline Constructos & CR & AVE & IMAGE & ACCES & EXPER & ATRACT & SERP & SERPAR & SATIS & COSTO & LEAL & RECO \\
\hline IMAGE & 0.861 & 0.5537 & $\mathbf{0 . 7 4 4 1 *}$ & & & & & & & & & \\
\hline ACCES & 0.9279 & 0.8115 & 0.2629 & $\mathbf{0 . 9 0 0 8}$ & & & & & & & & \\
\hline EXPER & 0.9047 & 0.7602 & 0.5144 & 0.2435 & $\mathbf{0 . 8 7 1 8} *$ & & & & & & & \\
\hline ATRACT & 0.871 & 0.7721 & 0.3102 & 0.0749 & 0.4073 & $\mathbf{0 . 8 7 8 6 *}$ & & & & & & \\
\hline SERP & 0.8783 & 0.5913 & 0.5656 & 0.3607 & 0.3724 & 0.2746 & $\mathbf{0 . 7 6 8} *$ & & & & & \\
\hline SERPAR & 0.9425 & 0.7666 & 0.265 & 0.1952 & 0.2763 & 0.0068 & 0.1877 & $\mathbf{0 . 8 7 5} *$ & & & & \\
\hline SATIS & 0.9524 & 0.8698 & 0.4238 & 0.3107 & 0.5745 & 0.2456 & 0.372 & 0.2631 & $\mathbf{0 . 9 3 2 6}$ & & & \\
\hline COSTO & - & - & 0.3347 & 0.2869 & 0.3255 & 0.1076 & 0.3338 & 0.2393 & 0.6303 & - & & \\
\hline LEAL & - & - & 0.2564 & 0.2224 & 0.3951 & 0.1468 & 0.2288 & 0.2832 & 0.6729 & 0.5977 & - & \\
\hline RECO & - & - & 0.3249 & 0.251 & 0.4617 & 0.2376 & 0.2756 & 0.3246 & 0.6559 & 0.5477 & 0.8034 & - \\
\hline
\end{tabular}

*/ Raíz cuadrada de AVE.

Fuente: Elaboración propia. 
La validez discriminante consta en evidenciar constructos que comparten más varianza con sus variables manifiestas que con otros constructos del modelo en cuestión. En este sentido, el cuadro 3 exhibe en la diagonal a valores de raíz cuadrada de AVE superiores a las cifras de la correlación de variables latentes que se encuentren a sus lados (Fornell y Larcker, 1981). Con este último paso, se ratifica el cumplimiento de manera satisfactoria de las pautas de evaluación del modelo de medidas, para enseguida continuar con la valoración del modelo estructural.

\subsubsection{Análisis del modelo estructural}

El análisis del modelo estructural abarca en gran medida la evaluación del nivel de significación de los coeficientes de regresión aplicando la técnica de bootstrap (Kline, 1998; Tenenhaus et al., 2005). Lo anterior se complementa con el examen de la pertinencia nomológica por medio de la $\mathrm{R}^{2}$ y la valoración de la calidad de las ecuaciones estructurales a través del coeficiente de Stone-Geisser $\mathrm{Q}^{2}$, ambos permiten evaluar la calidad predictiva del modelo interno (Chin, 1998; Tenenhaus et al., 2005). Esto va de la mano con la estimación del impacto de los constructos predictivos en los endógenos a través del examen del efecto relativo de tamaños $\left(f^{2}\right)$ y una evaluación de la bondad del ajuste (Gof) como una medida de calidad general de nuestros modelos de investigación.

La determinación del nivel de significación de las relaciones causales en ambos modelos se llevó a cabo calculando los valores de $t$ de Student con bootstrap de 500 remuestreos (Chin, 1998; Kline, 1998, Tenenhaus et al., 2005).

De un total de 11 relaciones entre variables latentes que componen cada uno de los dos modelos de estudio, seis relaciones resultaron significativas para el modelo A (todas con $\mathrm{P}<0.001)$ y ocho relaciones para el modelo $\mathrm{B}$ ( 6 con $\mathrm{P}<0.001$, una con $\mathrm{P}<0.01$ y otra con $\mathrm{P}<0.05)$. En el marco del mismo resultado, descartando la relación unidireccional entre COSTO y SATIS en el seno de los dos modelos de estudio, se observó que las cinco relaciones causales altamente significativas del modelo A son también altamente significativas en el modelo B. Este último modelo exhibe además dos relaciones significativas, una con $\mathrm{P}<0.01$ entre ACCES - $>$ SATIS y otra con P $<0.05$ entre SERP $->$ SATIS (Véase cuadro 4).

La calidad predictiva de los constructos endógenos pertenecientes a uno u otro de los dos modelos de estudio arroja, como se puede corroborar en el cuadro 5, valores de $\mathrm{R}^{2}$ superiores a la tasa admisible del $10 \%$ de la varianza explicada por la variación de las variables exógenas (Falk y Miller, 1992; Chin, 1998). En este sentido, tomando como referente a Wetzels et al., (2009), todos los constructos parecen expresar un amplio poder de predicción de sus respectivos modelos de estudio. No obstante, hay que recalcar que la $\mathrm{R}^{2}$ de la variable endógena SATIS registra una importante disminución pasando de 55.66 $\%$ en el modelo A al $38.66 \%$ en el modelo (B).

En consistencia con los resultados anteriores, el efecto relativo de tamaños $\left(f^{2}\right)$ evaluado según la guía de Cohen (1988) deja entrever un cierto paralelismo entre los dos modelos de investigación. Para el constructo SATIS, salvo el efecto moderado de EXPER y el amplio efecto del constructo COSTO en el modelo A, todos los demás están caracterizados por un efecto variable entre nulo y pequeño (véase cuadro 5). Para los dos constructos endógenos RECO y LEAL la similitud del $\left(f^{2}\right)$ en los dos modelos es perfecta: 


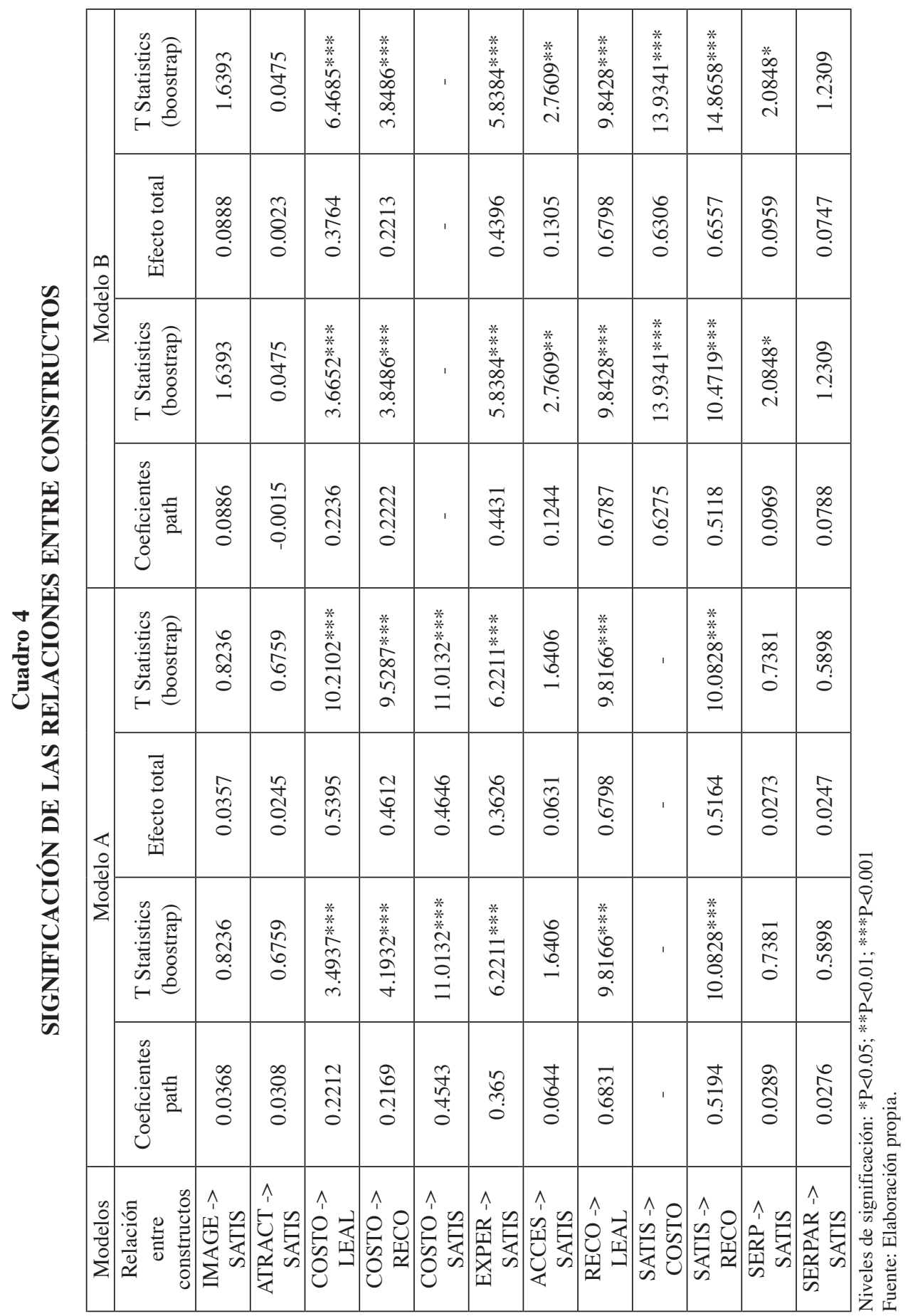


para RECO, SATIS tiene un amplio efecto y COSTO un efecto pequeño, mientras que para LEAL la variable RECO destaca un efecto amplio y COSTO un efecto moderado. Finalmente, el constructo COSTO tiene un impacto amplio en la variable SATIS.

En el cuadro 5 se exhibe también el nivel discriminatorio del índice de redundancia en la validación cruzada calculado con el coeficiente de Stone-Geisser $\left(\mathrm{Q}^{2}\right)$. Este último representa valores muy superiores a cero que confirman un alto poder predictivo para la estimación de las variables endógenas en ambos modelos de investigación, (Tenenhaus, 1999; Croutsche, 2009).

Finalmente, para la evaluación general de la calidad de los dos modelos de investigación se procedió al cálculo de la bondad del ajuste $(G o F)$, según el método de Tenenhaus et al., (2005), que arrojó un valor de 0.644 para el modelo A y 0.593 para el modelo B, indicando en ambos casos un $(G o F)$ superior a 0.36 , caracterizando así un amplio ajuste (Wetzels et al., 2009).

\section{Cuadro 5}

\section{PERTINENCIA DEL MODELO ESTRUCTURAL}

\begin{tabular}{|c|c|c|c|c|c|c|c|}
\hline \multicolumn{2}{|c|}{ Constructos } & \multicolumn{3}{|c|}{ Modelo A } & \multicolumn{3}{|c|}{ Modelo B } \\
\hline Endógenos & Predictivos & $\mathrm{R}^{2}$ & $\mathrm{Q}^{2}$ & $f^{2}$ & $\mathrm{R}^{2}$ & $\mathrm{Q}^{2}$ & $f^{2}$ \\
\hline \multirow{7}{*}{ SATIS } & IMAGE & \multirow{7}{*}{0.558} & \multirow{7}{*}{0.479} & 0.002 & \multirow{7}{*}{0.386} & \multirow{7}{*}{0.332} & 0.008 \\
\hline & ACCES & & & 0.009 & & & 0.024 \\
\hline & EXPER & & & 0.184 & & & 0.199 \\
\hline & ATRACT & & & 0.002 & & & 0.000 \\
\hline & SERP & & & 0.002 & & & 0.010 \\
\hline & SERPAR & & & 0.002 & & & 0.008 \\
\hline & COSTO & & & 0.390 & & & - \\
\hline \multirow{2}{*}{ RECO } & SATIS & \multirow{2}{*}{0.459} & \multirow{2}{*}{0.479} & 0.425 & \multirow{2}{*}{0.459} & \multirow{2}{*}{0.456} & 0.425 \\
\hline & COSTO & & & 0.054 & & & 0.054 \\
\hline \multirow{2}{*}{ LEAL } & RECO & \multirow{2}{*}{0.6812} & \multirow{2}{*}{0.669} & 0.406 & \multirow{2}{*}{0.681} & \multirow{2}{*}{0.668} & 0.406 \\
\hline & COSTO & & & 0.113 & & & 0.113 \\
\hline COSTO & SATIS & - & - & - & 0.397 & 0.398 & 0.661 \\
\hline
\end{tabular}

Fuente: Elaboración propia.

Del análisis del modelo de medidas y estructural de los dos modelos A y B se desprende la idea de una construcción teórica marcada por pocas diferencias y amplias similitudes que denotan pertinencia de la modelación y solidez predictiva. Es quizá en el análisis de estas diferencias que puede deslindarse el posicionamiento causal más idóneo para la interpretación de la relación calidad-precio en una modelación de la satisfacción turística. 


\section{RESULTADOS Y SU DISCUSIÓN}

Tomando en cuenta que tanto el tipo de investigación como los instrumentos empleados se caractericen por una abundancia de resultados, con la finalidad de centrarse en los objetivos del trabajo y darle dirección a esta discusión, hemos decidido enfocarnos en los hallazgos que más aportan a los propósitos inicialmente fijado a este ensayo. En el marco de esta dinámica de exposición, iniciaremos con la identificación de los alcances generales y seguir adelante con las particularidades que presenta cada uno de los dos modelos involucrados en este trabajo.

Independientemente de si se cuenta en otros contextos con antecedentes que corroboren una relación positiva entre la imagen del destino, sus atractivos y la satisfacción turística (Bigné et al., 2001; Chen y Chen, 2009; Chi y Qu, 2008), en este trabajo dicha relación parece relegada y sin efecto (Véase cuadro 4). En este sentido los constructos IMAGE y ATRACT además de su no significación en la valoración positiva de la satisfacción turística, se encuentren caracterizados por un efecto total bajo y no significativo en ambos modelos de investigación. Lo mismo ocurre con los servicios particulares (SERPAR), relacionados con una valorización de los entornos del turismo urbano (Elías Pastor, 2006), que no parecen exhibir un impacto significativo en la percepción positiva de la satisfacción turística. Estos hallazgos pueden ser producto de una motivación de viaje no centrada en la imagen, atractivos del destino y servicios particulares, traduciendo de esta manera un conjunto de relaciones causales no forzosamente factibles en todos los entornos turísticos.

En conformidad con lo observado en contextos descritos por Rial et al., (2008), los destinos bajacalifornianos, identificados -entre otros- por una degradada percepción de la seguridad pública (Bringas Rábago y Verduzco Chávez, 2008), no han logrado aún estructurar una imagen de marca capaz de impactar la satisfacción del turista. En el mismo orden de ideas, la motivación de viaje basada principalmente en la visita a familiares y/o amigos que caracteriza los cruces terrestres de los visitantes internacionales en la frontera bajacaliforniana no suele dejar una huella en la satisfacción al momento de evaluar atractivos y servicios de muy bajo consumo en este segmento (OTBC, 2013).

No obstante, para la accesibilidad del destino y los servicios públicos que constituyen aspectos importantes en la evaluación de la satisfacción turística (González Fernández y Esteban Talaya, 2000) estas relaciones se vuelven significativas solamente cuando la evaluación de la calidad-precio es considera un hecho posterior a la satisfacción turística (modelo B). En el marco de estas dos relaciones, la accesibilidad (ACCES) registra un mayor efecto total en la satisfacción turística comparado con los servicios públicos (SERP). Estos hallazgos pueden ser la traducción de expectativas de visita con menor sensibilidad a los precios y estas tienden más bien a centrarse en los motivos, medios y contextos de viaje (Wickens, 2002; Higgs et al., 2005). Estos últimos aspectos quizá ya se encuentran incorporados en el impacto de la experiencia de viaje (EXPER) en la satisfacción turística, que resulta en ambos modelos altamente significativa, siendo en el seno del modelo B en donde registra el efecto total más relevante de cualquiera de los constructos que definen la satisfacción (véase cuadro 4). En el marco de estos hallazgos, surge un destino turístico particular, diverso y complejo que parece responder a un modelo de satisfacción centrado en las experiencias de viaje definidas a partir de los intereses concretos del visitante (Richards, 2002). 
Desde la perspectiva estructural, la varianza explicada por el constructo de la satisfacción turística del modelo de investigación B refleja a un diferencial de $20 \%$ menos respecto al modelo A (véase cuadro 5). No obstante, en las condiciones del modelo A el efecto total de la calidad-precio en la satisfacción es altamente significativo con un valor de 0.4664 , mientras que en el modelo B el efecto total de la satisfacción en la calidadprecio es igual de significativo, ya que exhibe un valor de 0.6306. Estos resultados que expresan dos aspectos que parecen hasta cierto punto antagónicos, deben de tomarse en cuenta de manera concurrente para lograr un mejor entendimiento del alcance de las relaciones causales implicadas. El primer aspecto encuentra su expresión en el modelo A, reflejando un mayor poder explicativo de las diferencias observadas en la conformación del constructo de la satisfacción turística. Y el segundo que se desprende del modelo B, aunque indica una pertinente reciprocidad entre satisfacción y calidad-precio, la explicación de la calidad-precio por la satisfacción es más relevante. Aclarando que en ambos modelos los altos niveles de significación permiten hacer las mismas aseveraciones, por la naturaleza de los resultados anteriormente expuestos es quizás necesario subrayar que el modelo A se acomoda bien en una finalidad exploratoria, y el modelo B responde mejor a un interés predictivo.

En el contexto de los dos modelos de investigación la triangulación entre satisfacción (SATIS), recomendación (RECO) y lealtad (LEAL) al destino se caracteriza por una causalidad altamente significativa que llega a observarse también en sus vinculaciones respectivas con el constructo calidad-precio. En el marco de esta triangulación, los impactos totales registrados son también altos pero no parecen mostrar grandes diferencias entre los dos modelos. En este sentido, aunque los resultados del presente trabajo concuerden con lo observado por Yoon y Uysal (2005); Chi y Qu (2008); Yuksel et al. (2009); Cronin, et al. (2000); Rust, et al. (2000); Pedraja y Yagüe (2004), el impacto total de la satisfacción en la recomendación es del orden de 0.14 a favor del modelo B y en lo que toca al impacto de la recomendación en la lealtad las cifras son idénticas en ambos modelos (véase cuadro 4). En conjunto, estos hallazgos indican que la consideración de la calidad-precio como antecedente de la satisfacción o como su consecuencia no parece afectar el comportamiento intencional del visitante que consiste en la lealtad al destino y su recomendación a conocidos y familiares. Estos mismos resultados se encuentren reconfortados en el marco del análisis del efecto relativo de tamaños $\left(f^{2}\right)$ que viene caracterizado por valores similares en lo que toca a la recomendación y lealtad, además para la recomendación parece destacarse la satisfacción con un amplio efecto mientras que para la lealtad encuentra su efecto amplio en la recomendación (véase cuadro 5). Del mismo modo, como se corrobora en otros estudios (Truong y Foster, 2006), vistas desde la perspectiva de la evaluación calidad-precio estas relaciones causales resultaron altamente significativas en ambos modelos, aunque su efecto total es más relevante en la lealtad del visitante y considerablemente más importante en el modelo A, tanto para la disposición a recomendar el destino como para reforzar la lealtad (Véase cuadro 4).

La reciprocidad que parece caracterizar en ambos modelos a la relación entre calidadprecio y satisfacción del visitante abra para la reflexión y la acción la posibilidad de considerar una revisión de los precios aplicados en el destino a favor de la oferta. Lo anterior cobra un especial interés si se toma en cuenta el abordaje de una eficiencia que ha sido 
sinónimo de un crecimiento con tensión distributiva entre los diferentes grupos sociales que componen a las comunidades dedicadas a la actividad turística (Copeland, 1991). Desde luego, en estos contextos proclives a la demanda la sola mención de una posibilidad de revisión del precio a favor de la oferta es suficiente para encender a un apasionante debate económico que será probablemente concluido a favor de la tradicional consolidación de la competitividad por los precios (Masiero y Nicolau, 2012). Pero para el campo de la gestión y mercadotecnia estirar los precios a favor de la oferta no parece extraño, ni descabellado si este interés se encuentra planteado para conservar o en su caso mejorar los niveles de satisfacción del consumidor (Gijsbrechts, 1993; Dolcinar 2004, Masiero y Nicolau, 2012). De hecho, independientemente de un pragmatismo económico que nos ha acostumbrado a una visión centrada generosamente en la demanda, la eventualidad de un estiramiento de los precios se contempla en un planteamiento distributivo que permite tomar en cuenta la demanda, los actores y el contexto de estructuración de la oferta. En este sentido, el abordaje desde la perspectiva de la eficiencia que ha sido propio de un turismo concebido como generador de crecimiento no ha dejado de alimentar a una tensión distributiva entre los diferentes grupos sociales que componen a las comunidades que se dedican a este tipo de actividades (Copeland, 1991).

\section{CONCLUSIONES}

El presente trabajo enfocado en determinar el impacto de la calidad-precio en la satisfacción turística, la disposición a recomendar el destino y la lealtad del visitante, más allá de sus propios hallazgos, evidencia la dificultad de reconciliar lo conocido con lo esperado de la modelación teórica en un destino turístico particular, diverso y complejo.

En esta investigación se confirma una incidencia recíproca, altamente significativa, de la evaluación de la calidad-precio por el visitante internacional terrestre en la satisfacción turística. En este hallazgo, la evaluación calidad-precio parece un constructo de modelación factible tanto como antecedente (modelo A) o consecuencia de la evaluación de la satisfacción turística (modelo B). No obstante, solo con la consideración de la calidadprecio como un hecho posterior a la satisfacción turística (modelo B) se logra un impacto estadísticamente aceptable de los demás aspectos inherentes a la satisfacción (accesibilidad y servicios públicos). De esta manera, lo anterior queda plasmado conceptualmente en los límites de una satisfacción turística centrada en la motivación concreta del visitante.

De igual manera que en otros estudios, los impactos observados entre satisfacción, recomendación y lealtad resultaron para ambos modelos altamente significativos en un marco vinculante con la calidad-precio. Esto parece indicar que la inserción de la calidadprecio como antecedente o resultado de la satisfacción no afecta significativamente el comportamiento intencional del visitante para regresar y/o recomendar el destino. En este contexto, los efectos totales más relevantes se registran entre la recomendación y la lealtad al destino, mientras que entre la satisfacción y la recomendación, el impacto más alto se identifica con el modelo B cuando la calidad-precio es resultante de la satisfacción del visitante. Estos hallazgos dejen a la oferta turística en el destino un margen de maniobra que permite una gestión a su favor de la calidad-precio cuando es resultante de una satisfacción turística que termina impactando en el comportamiento intencional del visitante. 
Enfocando las relaciones de la calidad-precio con recomendación y lealtad, éstas además de caracterizarse por una alta significación, exhiben un efecto total más relevante en el modelo A. En el marco de las diferencias que pueden llegar a destacar en la estructuración causal entre calidad-precio y satisfacción turística, se mencionará una mayor disposición del modelo A para cumplir con una finalidad exploratoria para la consolidación teórica, mientras el modelo B se encamina más bien hacia un objetivo predictivo en un campo empírico concreto. Siendo ambas modelaciones de carácter estadísticamente factible, esta posible dualidad que puede llegar a plantearse en una interpretación secundaria, nos permite subrayar una vez más la complejidad de lograr un marco unificado aplicado universalmente a la satisfacción turística.

Con relación al impacto reciproco de la calidad-precio en la satisfacción, este hallazgo abra a la reflexión y la praxis de la gestión y mercadotecnia una alternativa para estirar los precios a favor de los actores de la oferta. En este sentido, replantear los términos de la relación entre eficiencia de la actividad turística y la distribución equitativa de sus beneficios será traducido en el reto fundamental de mejorar las condiciones de la oferta sin por lo tanto, descuidar la percepción positiva del visitante.

\section{BIBLIOGRAFÍA}

AINSCOUGH, T. (2005): «The effect of brand, agent, and price on consumer evaluation of travel services», Journal of Travel \&Tourism Management, vol. 19 (1), pp. 39-48.

ALBACETE-SAEZ, C.A., FUENTES-FUENTES, M.M. y LLORÉNS-MONTES, F.J. (2007): «Service quality measurement in rural accommodation», Annals of Tourism Research, vol. 34 (1), pp. 45-65.

ALEGRE, J. y JUANEDA, C. (2006). «Destination loyalty-consumer's economic behavior», Annals of Tourism Research, vol. 33 (3), pp. 684-706.

BAGOZZI, R.P. (1994): «Structural equation models in marketing research: basic principles», In Bagozzi, R.P. (Ed.), Basic Principles of Marketing Research. Oxford: Blackwell, pp. 317-385.

BAKER, D.A. y CROMPTON, J.L. (2000): «Quality, satisfaction and behavioral intentions», Annals of Tourism Research, vol. 27 (3), pp. 785-804.

BARCLAY, D., HIGGINS, C. y THOMPSON, R. (1995): «The Partial Least Squares (PLS) Approach to Causal Modelling: Personal Computer Adoption and Use as an Illustration», Technology Studies, vol. 2 (2), pp. 285-309.

BIGNÉ, J.E. y ANDREU L. (2004): «Modelo cognitivo-afectivo de la satisfacción en servicios de ocio y turismo», Cuadernos de Economía y Dirección de Empresa, (21), pp. 89-120.

BIGNÉ, J.E., SÁNCHEZ, M.I. y SÁNCHEZ, J. (2001): «Tourism image, evaluation variables and after purchase dimensions». Tourism Management, vol. 22, pp. 127-133.

BONET AGUSTI, L.L. (2008): «Ciudad, cultura y modelos turísticos». Metropolis, (72), pp. 66-69.

BOSQUE, I. y MARTÍN, H. (2008): «Tourist satisfaction: A cognitive-affective model». Annals of Tourism Research, vol. 35, (2), pp. 551-573. 
BRINGAS RÁBAGO, N.L. y VERDUZCO CHÁVEZ, B. (2008): «La construcción de la frontera norte como destino turístico en un contexto de alertas de seguridad». Región y Sociedad, vol. 42, pp.3-36.

BURNETTE, J.L. y WILLIAMS, L.J. (2005): «Structural Equation Modeling (SEM): an introduction to basic techniques and advanced issues». In Swanson, R.A. y Holton, E.F. (Eds.), Research in organization: foundations and methods of inquiry, San Francisco: Berret-Koehler Publishers, pp. 143-160.

CAMPO, S. y YAGÜE, M.J. (2009): «Análisis de la influencia del precio en la formación de la satisfacción: una aplicación al mercado turístico». Revista Española de Investigación de Marketing ESIC, vol. 13 (2), pp. 31-52.

CASTAÑO BLANCO, J.M., MORENO SÁEZ, A., GARCÍA DAUDER, S. y CREGO DÍAZ, A. (2003): «Aproximación psicosocial a la motivación turística: variables implicadas en la elección de Madrid como destino», Estudios Turísticos, n ${ }^{\circ} 158$, pp. 5-41.

CENTRO DE ESTUDIOS SUPERIORES DEL TURISMO (CESTUR) (2011),:Metodología de estudio de perfiles y satisfacción del turista, Secretaria de Turismo, México.

CHEN, C.F. y CHEN, F.S. (2009): «Experience quality, perceive value, satisfaction and behavioral intentions for heritage tourists», Tourism Management, vol. 31 (1), pp. 29-35.

CHI, C. G.Q. y QU, H. (2008): «Examining the structural relationships of destination image, tourist satisfaction and destination loyalty: An integrated approach». Tourism Management, vol. 29 (4), pp. 624-636.

CHIN, W.W. (1998): «The partial Least squares approach to structural equation modeling», In Marcoulides, G.A. (Ed), Modern methods for business research, Mahwah: Lawrence Erlbaum Associates Publisher, pp. 295-336.

CHURCHILL, G.A. y SURPRENANT, C. (1982): «An investigation into the determinants of customer satisfaction», Journal of Marketing Research, vol. 19, pp. 491-504.

COHEN, J. (1988), Statistical Power Analysis for the Behavioral Sciences, Lawrence Erlbaum Associates, Hillsdale.

COPELAND, B.R. (1991): «Tourism, welfare, and de-industrialization in a small open economy». Economica, vol. 58, pp. 515-529.

CRONIN, J.C. y TAYLOR, S.A. (1992): «Measuring service quality: a re-examination and extension», Journal of Marketing, vol. 56 (3), pp. 55-68.

CRONIN, J.J., BRANDY, M.K. y HULT, G.T.M. (2000): «Assessing the effects of quality, value and customer satisfaction on consumer behavioral intentions in service environments», Journal of Retailing, vol. 76 (2), pp.193-218.

CROUTSCHE, J.J. (2009): Analyse des données en Marketing, en Management et en Sciences Sociales: Conceptualisation et Applications, Paris: Editions ESKA.

DEVESA, M. y PALACIOS, A. (2005): «Predicciones en el nivel de satisfacción percibida por los turistas a partir de variables motivacionales y de valoración de la visita», Información Comercial Española, $\mathrm{n}^{\circ}$ 821, pp. 241-255.

DIAMONTOPOULOS, A. y WINKLHOFER, H.M. (2001): «Index construction with formative indicators: an alternative to scale development», Journal of Marketing Research, $\mathrm{n}^{\circ} 38$, pp. 269-277. 
DODDS, W.B., MONROE, K.B. y GREWAL, D. (1991): «Effects of price, brand, and store information on buyers' product evaluations», Journal of Marketing Research, vol. 28 (3), pp. 307-318.

DOLNICAR, S. (2004): «Beyond 'commonsense segmentation': a systematics of segmentation approaches in tourism.» Journal of Travel Research, vol. 42, pp. 244-50.

ELÍAS PASTOR, L. (2006): El Turismo del Vino. Documentos de Estudios de Ocio, (30), Bilbao: Publicaciones de la Universidad de Deusto.

FALK, R.F y MILLER, N.B. (1992): A primer for soft modeling, The University of Akron, Akron.

FORNELL, C. y LARCKER, D.F. (1981): «Evaluating Structural Equation Models with Unobservable Variables and Measurement Error», Journal of Marketing Research, vol.18, pp. 39-50.

FORNELL, C. y WERNERFELT, B. (1987: «Defensive marketing strategy by customer complaint Management», Journal of Marketing Research, vol. 24 , pp. 337-346.

FORNELL, C. (1992): «A National Customer Satisfaction Barometer: The Swedish Experience», Journal of Marketing, vol. 56 (1), pp. 6-21.

GIJSBRECHTS, E. (1993): «Prices and pricing research in consumer marketing: some recent developments.» International Journal of Research in Marketing, vol.10, pp.11551.

GONZÁLEZ FERNÁNDEZ, A. y ESTEBAN TALAYA, A. (2000): Valores y estilos de vida en el análisis de la demanda turística. Feria Internacional de Turismo.

GUNDERSEN, M.G. y OLSSON, U.H. (1996): «Hotel guest satisfaction among business travellers: What are the important factors? The Cornell Hotel and Restaurant», Administration Quarterly, vol. 37 (2), pp. 72-81.

GUPTA, S., LEHMANN, D., y STUART, J. (2004): «Valuing customer», Journal of Marketing Research, vol. 41 (1), pp. 7-18.

HALSTEAD, D., HARTMAN, D. y Schmidt, S.L. (1994): «Multisource Effects on the satisfaction Formation Process» Journal of the Academy of Marketing Science, vol. 22, pp. 114-129.

HASEGAWA, H. (2010): «Analyzing tourists' satisfaction: A multivariate ordered probit approach», Tourism Management, vol. 31 (1), pp. 86-97.

HENSELER, J., RINGLE, C.M. y SINKOVICS, R.R. (2009): «The use of partial least squares path modeling in international marketing», In: Sinkovics, R.R. y Ghauri, P.N. (Eds.), Advances in International Marketing, Bingley: Emerald Group Publishing Limited, pp. 277-319.

HIGGS, B., POLONSKY, M.J. y HOLLICK, M. (2005): «Measuring Expectations: Forecast vs. Ideal Expectations. Does It Really Matter?», Journal of Retailing and Consumer Services, vol. 12, pp. 49-64.

HSU, H.H., CHEN, W.H. y HSIEH, M.J. (2006): «Robustness testing of PLS, LISREL, EQS and ANN-based SEM for measuring customer satisfaction», Total Quality Management and Business Excellence, vol. 17 (3), pp. 355-372.

JÖRG, H., RINGLE, C., SINKOVICS, M. y RUDOLF, R. (2009): «The use of partial least squares path modeling in international marketing». Advances in International Marketing, vol. 20, pp. 277-319, 
KIM, W.G., NG, C.Y.N. y KIM, Y.S. (2009): «Influence of institutional DINESERV on customer satisfaction, return intention, and word-of-mouth», International Journal of Hospitality Management, vol. 28, pp. 10-17.

KLINE, R.B. (1998): Principles and Practice of Structural Equation Modeling. New York: The Guilford Press.

KOZAC, M. y RIMMINGTON, M. (2000): «Tourist satisfaction with Mallorca, Spain, as an off-season holiday destination», Journal of Travel Research, vol. 38 (3), pp. 260-269.

LAGUNA, M. y PALACIOS, A. (2009): «La calidad percibida como determinante de tipologías de clientes y su relación con la satisfacción: Aplicación a los servicios hoteleros». Revista Europea de Dirección y Economía de la Empresa, vol. 18 (3), pp. 189-212.

MASIERO, L y NICOLAU, J.H. (2012): «Tourism market segmentation based on price sensitivity: finding similar price preferences on tourism activities». Journal of Travel Research, vol. 51 (4), pp. 426-435.

MCKNIGHT, D.H., CHOUDHURY, V. y KACMAR, C. (2002): «Developing and validating trust measures for e-commerce: an integrative typology», Information Systems Research, vol. 13 (3), pp. 334-359.

MOON, S., RUSSELL, G.J. y DUVVURI, S.D. (2006): «Profiling the reference price consumer», Journal of Retailing, vol. 82 (1), pp.1-11.

NUNNALLY, J.C., BERNSTEIN, I.H. (1994): Psychometric Theory, 3rd ed. New York: McGraw-Hill.

OBSERVATORIO TURÍSTICO DE BAJA CALIFORNIA (OTBC) (2013): Caracterización de los visitantes internacionales en las ciudades fronterizas de Baja California: Mexicali, Tecate y Tijuana (verano 2013), El Colegio de la Frontera Norte-Secretaría de turismo del estado de Baja California, Tijuana.

OPPERMANN, M. (2000): «Tourism Destination Loyalty», Journal of Travel Research, vol. 39 (1), pp. 78-84.

PEDRAJA, M. y YAGÜE, M.J. (2004): «Perceived quality and price: their impact on the satisfaction of restaurant customers». International Journal of Contemporary Hospitality Management, vol. 16 (6), pp. 373-379.

PENG, D.X. y LAI, F. (2012): «Using partial least squares in operations management research: A practical guideline and summary of past research», Journal of Operations Management, vol. 30, 467-480.

PETRICK, J. (2004): «First timers' and repeaters' perceived value», Journal of Travel Research, vol. 43 (1), pp. 29-38.

RAO, A.R. y MONROE, K.B. (1988): «The moderating effect of prior knowledge on cue utilization in product evaluations», Journal of Consumer Research, vol. 15, pp. 253-261.

REICHHELD, F.F. y SASSER, W.E. (1990): «Zero defections: quality comes to services», Harvard Business Review, vol. 68, pp.105-111.

REICHHELD, F.F. (1996): The Loyalty Effect. Boston: Harvard Business School Press.

RICHARDS, G. (2002): «Tourism attraction systems, exploring cultural behavior», Annals of Tourism Research, vol. 29 (4), pp.1048-1064.

RIAL, A., GARCÍA, A. y VARELA, J. (2008): «Una aplicación metodológica para el estudio de la imagen de marca en un destino turístico», Revista de Turismo y Patrimonio Cultural, vol. 6 (1), pp. 1-11. 
RUST, R.T., DANAHER, P. y VARKI, S. (2000): «Using service quality data for competitive marketing decisions», International Journal of Service Industry Management, Vol. 11 (5), pp. 438-469.

SHOEMAKER, S. y LEWIS, R.C. (1999): «Customer loyalty: the future of hospitality marketing», International Journal of Hospitality Management, vol. 18, pp. 345-370.

SÖDERLUND, M. (1998): «Customer satisfaction and its consequences on customer behavior revisited. The impact of different levels of satisfaction on word-of-mouth, feedback to the supplier and loyalty», International Journal of Service Industry Management, vol. 9 (2), pp. 169-188

TENENHAUS, M., ESPOSITO VINCI, V., CHATELIN Y.M. y LAURO C. (2005): PLS Path Modeling, Computational Statistics and Data Analysis, (28), pp. 159-205.

TENENHAUS, M. (1999): «L'approche PLS». Revue de Statistique Appliquée, vol. 47 (2), pp. 5-40.

TRUONG, T. y FOSTER, D. (2006): «Using HOLSTAT to evaluate tourist satisfaction at destinations: The case of Australian holidaymakers in Vietnam». Tourism Management, vol. 27 (5), pp. 842-855.

VOSS, G.B., PARASURAMAN, A. y GREWAL, D. (1998): «The roles of price, performance and expectations in determining satisfaction in services exchanges», Journal of Marketing, vol. 62 (4), pp. 46-61.

WANG, X., ZHEN, F., ZHANG, J. y WU, X. (2014): «Exploring Factors Influencing Residents' Satisfaction with Tourism Development in Poverty-Stricken Areas: A Case Study of Five Poor Villages in China, Asia Pacific Journal of Tourism Research, vol. 19 (5), pp. 517-537.

WETZELS, M., ODEKERKEN-SCHRÖDER, G. y VAN OPPEN, C. (2009): «Using PLS path modeling for assessing hierarchical construct models: Guidelines and empirical illustration». MIS Quarterly, vol. 33 (1), pp. 177-195.

WICKENS, E. (2002): «The sacred and the profane. A tourist typology», Annals of Tourism Research, vol. 29, pp. 834-851.

YADAV, M.S. y SEIDERS, K. (1998): «Is the price right? Understanding contingent processing in reference price formation», Journal of Retailing, vol. 74 (3), pp. 311-329.

YOO, D.K. y PARK, J.A. (2007): «Perceived service quality: analyzing relationships among employees, customers, and financial performance». International Journal of Quality \& Reliability Management, vol. 24 (9), pp. 908-926.

YOON, Y. y UYSAL, M. (2005): «An examination of the effects of motivation and satisfaction on destination loyalty: a structural model», Tourism Management, vol. 26 (1), pp. 45-56.

YUKSEL, A., YUKSEL, F. y BILIM, Y. (2009): «Destination attachment: Effects on customer satisfaction and cognitive, affective and conative loyalty». Tourism Management, vol. 31 (2), pp. 274-284.

ZEITHAML, V.A., BERRY, L.L., y PARASURAMAN, B.A. (1996): «The behavioral consequences of service quality», Journal of Marketing, vol. 60, pp. 31-46.

ZEITHMAL, V.A. (1988): «Consumer perceptions of price, quality and value: a meansend model and synthesis of evidence», Journal of Marketing, vol. 52, pp. 2-22. 
